\title{
Recurrent Transvaginal Leak of Dialysate as a Complication of Peritoneal Dialysis Catheter Tip Capture by Fallopian Tube after Tubal Occlusion
}

\author{
Maha A Hummeida ${ }^{a^{*}}$, Sara E Khalid ${ }^{a}$, Mohammed H A Modawi ${ }^{a}$, Aymun I Mobarak ${ }^{\mathrm{a}}$, Abdel-Mutalab \\ M Imam ${ }^{\mathrm{b}}$, Hisham Elbadawic, Hasan Abu-Aisha ${ }^{\mathrm{a}}$
}

a. Ribat Center for Peritoneal Dialysis, Ribat University Hospital, Sudan

b. Department of Obstetrics and Gynecology, Soba Teaching Hospital, Sudan

c. Department Of Radiology, Ribat University Hospital, Sudan

\begin{abstract}
Introduction: Peritoneal dialysis(PD) cathetermalfunction may result from obstruction by various intra-peritoneal structures. Such obstruction may rarely be caused by fallopian tubes, in which case it may present as out flow failure and/or per vaginum (PV) leak of dialysate.
\end{abstract}

Case report: A 46 years old female developed early out-flow obstruction while maintaining adequate dialysate inflow soon after PD catheter insertion. The patient also had PV leak of the dialysate that was misinterpreted by the patient as urine incontinence. Fluoroscopic catheterogram confirmed catheter patency and outlined the right fallopian tube. Magnetic Resonance Imaging (MRI) revealed the presence of fluid in vagina with no obvious peritoneovaginal fistulous track. Laparoscopy was performed, at which time examination under anesthesia excluded the presence of peritoneo-vaginal fistula. During laparoscopy, the catheter tip was found to be captured by the fimbriae of the right fallopian tube. The catheter tip was released and right tubal occlusion was performed, after which adequate flow was restored. Three month later, the catheter was replaced because of fungal peritonitis. Vaginal leak recurred six weeks after insertion of the new catheter through the same occluded tube. Mini laparotomy and tubal ligation with catheter removal were done and the patient was maintained on hemodialysis until she received a kidney transplant four months later.

Conclusion: Vaginal leak of dialysate secondary to fallopian tube capture of PD catheter tip can be treated laparoscopically. It is important to distinguish between this and peritoneo-vaginal fistula which often requires laparotomy for fistulous tract debridement and repair.

\footnotetext{
* Corresponding author; Ribat Center for Peritoneal Dialysis, Ribat University Hospital, Burri, P.O. Box 55, Khartoum, Sudan.

E mail: mahoyaali123@hotmail.com
}

Keywords: Catheter Malfunction; Fallopian Tube; Peritoneal Dialysis; Transvaginal leak

\section{The authors declared no conflict of interest}

\section{Introduction}

Peritoneal dialysis (PD) is a well recognized modality of renal replacement therapy (RRT) that hinges on a properly functioning dialysis catheter. Catheter malfunction remains a significant cause of technique failure. Common causes of malfunction include catheter displacement, omental or bowl wrapping and fibrin clots. Less commonly, various intraperitoneal structures have been anecdotally reported to cause catheter obstruction such as the appendix, appendices/epiploicae of the sigmoid colon and fallopian tubes [1]. In case of PD catheter obstruction by fallopian tube, the presentation is usually out-flow difficulties, edema of the anterior abdominal wall and external genitalia [2] and per vaginum (PV) leak of the dialysis fluid. Interestingly the latter was misdiagnosed as urinary stress incontinence in four out of the six reported cases [1]. PV leak may occur as an early complication, usually secondary to entrapment or capture of PD catheter into the fallopian tube fimbriae, or as a late event resulting from peritoneo-vaginal fistula [2]. Recurrent polymicrobial peritonitis (suggestive of a gynecological source) and fungal peritonitis in a female patient can be the presenting symptom of abnormal communication between the peritoneum and vagina rather than overt vaginal leak [3].

\section{Case report}

A 46 years old female with end stage renal disease (ESRD) of unknown etiology started continuous ambulatory peritoneal dialysis (CAPD) as RRT after difficulties in maintaining hemodialysis (HD) vascular access. The patient was multiparous with no significant gynecological 
Figure 1: Plain abdominal radiograph showing the pelvic location of the peritoneal dialysis catheter tip which appears to be compressed

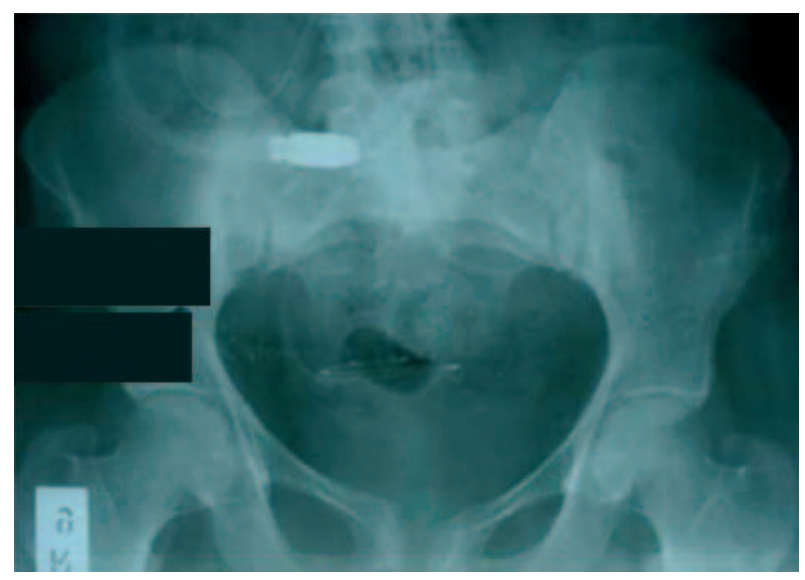

history apart from a cesarean section. The PD access was achieved through a surgically inserted double-cuff Tenckhoff coiled catheter. PD was commenced after two weeks but there was out-flow obstruction that did not respond to conservative measures. A plain abdominal radiograph revealed that the catheter was located deep in the pelvis, but the coiled part appeared to be compressed or trapped into a compartment (Figure 1). The presence of adhesions or omental wrapping was considered because the patient had history of emergency cesarean section 23 years before. Intraluminal instillation of streptokinase was contemplated, and she was asked about the presence of vaginal bleeding that will contraindicate its administration. She denied any vaginal discharge or leak but admitted to urinary incontinence that she experienced a few minutes after dialysate instillation. A vaginal examination demonstrated the drainage of clear fluid which proved to be the dialysate with its high glucose concentration. Subsequently, a dye-enhanced catheterogram showed a patent catheter and the contrast outlined a tortuous structure in the right pelvic cavity, supposedly the right fallopian tube. The contrast also appeared in the vagina at the end of the study although it didn't delineate the uterus (Figure 2). Peritoneo-vaginal fistula and catheter tip capture by fallopian tube were considered to be possible explanations. To verify that impression we performed a hysterosalpingogram (HSG) which demonstrated a smooth uterus; the left fallopian tube was normal while the right was dilated with contrast leaking through it freely into peritoneal cavity (Figure 3). Furthermore, a magnetic resonance image scan (MRI) with 500 milliliter of dialysate with contrast showed fluid in the vagina and uterus. Although there was no obvious direct communicating track between the peritoneal cavity and vagina, a peritoneo-vaginal fistula could not be excluded (Figure 4). This is an uncommon and usually
Figure 2: Catheterogram shows patent catheter and the contrast outlining a tortuous structure (arrowed) in the right pelvic cavity, likely the right fallopian tube

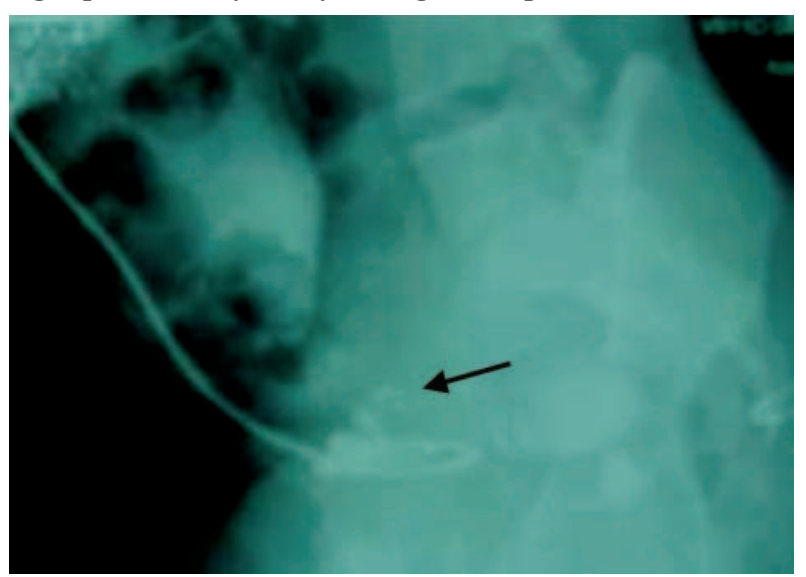

late complication of CAPD that has been described to occur secondary to erosion of the vaginal wall by the catheter tip. The second possibility of catheter capture by the fallopian tube seemed to be more reasonable as it is more likely to occur as an early CAPD complication.

Given what has been described above we decided to perform an exploratory laparoscopy that revealed the absence of a fistula. However, omental adhesions along the catheter down to the right fallopian tube was observed and the catheter tip was found to be buried within the adhesions and captured by the fimbirae of a dilated right fallopian tube. The uterus was covered with massive adhesions, and the left ovary and its tube were adherent to the pelvic wall as well. The catheter was released and placed away from the tube. Tubal occlusion was performed using bipolar diathermy. Immediate postoperative out-flow was excellent with no more vaginal leak.

PD was restarted on the second day, and she did well for three months after which she developed fungal peritonitis caused by Candida albicans. The catheter was removed as part of the management of this infection. On the background of HD access difficulties and patient desire we decided to maintain the patient on PD via a temporary single cuff Tenckhoff catheter that was concurrently inserted when the original catheter was removed. Six week later, she developed transvaginal leak again. A catheterogram showed the catheter tip captured by the same right fallopian tube. A mini-laparotomy was arranged on the background of the previously noted extensive adhesions. Again, substantial pelvic adhesions involving the fallopian tubes and the catheter were noted. The catheter was released and removed, and bilateral tubal ligation was consequently performed. This time the 
Figure 3: Hysterosalpingogram demonstrating dilated right fallopian tube with contrast leaking through it into the peritoneal cavity

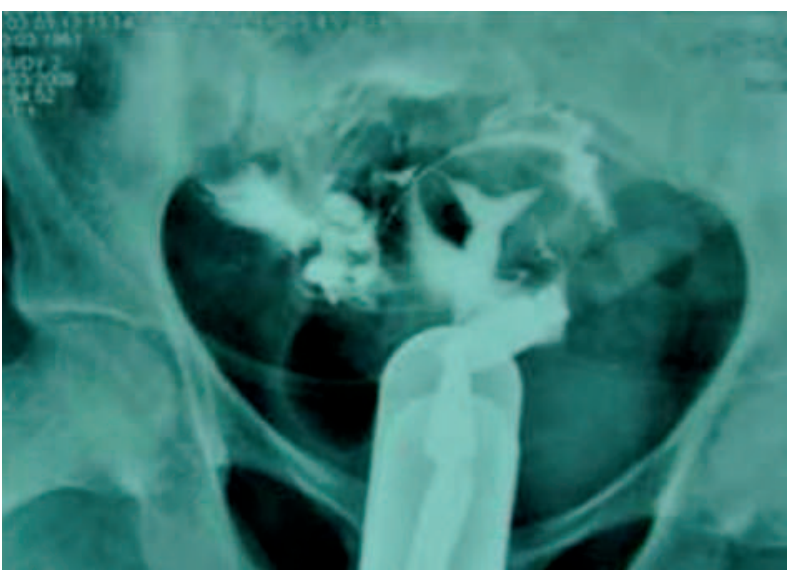

patient refused PD; she was maintained on HD until she underwent renal transplantation four months later.

\section{Discussion}

The fallopian tubes are the natural communicating tract between the peritoneal cavity and genital system in females. Anatomically, the epithelium of the fallopian tubes merges with that of peritoneum and the dialysate fluid cannot gain access through this tract unless it overcomes the high resistance in the fallopian tubes [4]. This can occur when the PD catheter tip gets captured by the omentum or fallopian fimbirae soon after insertion, generating a high flow that can counteract the resistance in the fallopian tube resulting in transvaginal leakage.

Vaginal leakage of dialysate can occur as an early complication of CAPD usually secondary to fallopian tube capture of PD catheter tip. It may also be a late complication as a result of perforation by catheter tip creating a peritonio-vaginal fistula. Such perforation may be caused by direct pressure necrosis $[5,6]$, or as a consequence of malnutrition or peritonitis. Peritonitis episodes may weaken the visceral wall and facilitate pressure necrosis, directly via the inflammatory process itself, or indirectly by the negative effect on the nutritional status of the patient [7]. Catheter tip capture by Fallopian tube can also occur as a late complication of PD [4] and this has been attributed to peritonitis causing omental and visceral wrapping that brings the tip close to the fallopian tube.

Peritonitis can be a causative factor for transvaginal leak as described above or vice versa. Retrograde passage of infected material during menstruation [3] and salpingitis $[3,8]$ can be predisposing factors for peritonitis. This is usually of the recurrent type caused by polymicrobial
Figure 4: Magnetic Resonance Image (MRI): contrast shows fluid in the vagina and uterus with no obvious fistulous track between peritoneal cavity and vagina

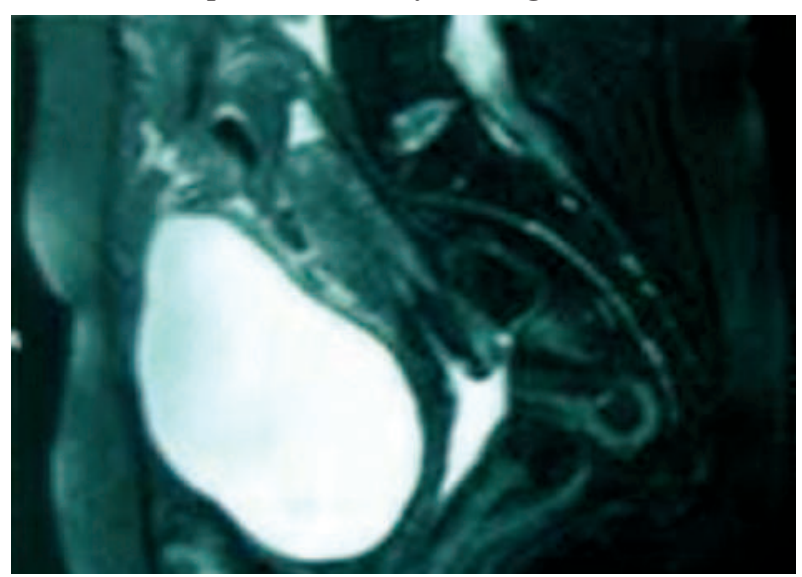

organisms suggestive of gynecological sources [3] or fungal peritonitis $[3,5,9]$. Therefore, these types of peritonitis in female CAPD patients, especially with no usual predisposing factors such as previous antibiotic therapy, diabetes mellitus or immunosuppressive treatment, should raise suspicion of transvaginal leak. Such leak may interestingly be misdiagnosed as urinary stress incontinence [1]. It can also be completely missed because the patient may pass it as vaginal discharge or refrain from mentioning it.

Klein et al reviewed ten cases of PD catheter complications caused by fallopian tubes. In six cases the capture occurred in the right tube, as in our case. The presenting features were outflow obstruction, urine incontinence and vaginal discharge [1].

Clinical diagnosis of transvaginal leak can simply be verified by intraperitoneal injection of methylene blue or testing the discharged fluid for glucose. Additional imaging tests are required to diagnose the cause; whether fallopian tube capture or peritone-ovaginal fistula. This is important because in the former, laparoscopy is frequently needed to free the tube. Concurrent tubal ligation or salpingectomy (if fertility is not an issue) are the definitive treatment, with excellent long term outcome and early resumption of PD within 3 days after temporary HD [1]. However, in the latter case, laparotomy is more often needed than not for fistulous tract debridement and repair, and RRT usually needs to be maintained by temporary HD for a while before restarting PD [5, 6, 9].

Different complementary diagnostic tests are available with different yielding results. Catheterogram is a simple test and can exclude kinking or obstruction, confirm the patency of the catheter and show the direct communication between the peritoneal cavity and genital 
tract via the fallopian tube $[4,6]$. Hysterosalpingogram [9] can demonstrate the fistula tract or the communication between genital system and peritoneal cavity as in our case. Bradley et al reported the first case of transvaginal leak caused by erosion that was demonstrated by computed tomography peritoneaography (CTP) [5]. Exploratory laparotomy has been used as a diagnostic and therapeutic procedure after clinical confirmation of vaginal leak of dialysate [2, 8]. Exploratory laparoscopy could replace laparotomy if entrapment or capture was suspected [1].

In our case, the laparoscopic tubal occlusion using diathermy resolved the problem completely. The catheter functioned well with no more vaginal leak until it was replaced by a temporary one three months later as a part of the management of fungal peritonitis. The development of fungal peritonitis can be explained by partial or incomplete tubal occlusion creating a narrow segment of fallopian tube lumen that can still permit passage of organisms but prevent fluid leak, particularly after the catheter tip has been shifted away from the fallopian tube. The recurrent vaginal leak may have occurred as a result of high flow pressure from the new catheter tip which lied down close to the fallopian tube. Moreover, the fallopian tube might have captured the catheter as a consequence of the inflammatory process and adhesions caused by peritonitis, as verified in the mini-laprotomy. This high flow may gradually re-canulate and dilate the narrow segments of partially occluded fallopian tube to the extent that allowed fluid leak. As illustrated in this case, we believe that laparoscopy represents a good tool for diagnosing and treating PD catheter obstruction by fallopian tubes and vaginal leak.

\section{Conclusion}

Vaginal leak of dialysate can occur as an early complication of PD secondary to fallopian tube capture of PD catheter tip. It is important to distinguish between tubal capture of the catheter tip which can be treated laparoscopically and peritoneo-vaginal fistula which often requires laparotomy for fistulous tract debridement and repair.

\section{References}

1. Klein Z, Magen E, Fishman A, Korzets Z. Laparoscopic Salpingectomy: The definitive treatment for peritoneal dialysis catheter outflow obstruction caused by oviductal fimbriae. J Laparoendosc Adv Surg Tech A. 2003 Feb;13(1):65-8.

2. Caporale N,PerezD, Alegre S. Vaginal leak of peritoneal dialysis liquid. Perit Dial Int. 1991;11(3):284-5.

3. Swartz RD, Campbell DA, Stone D, Dickinson C. Recurrent polymicrobial peritonitis from a gynecologic source as a complication of CAPD. Perit Dial Int. $1983 ; 3: 32-3$.

4. Macallister RJ, Morgan SH. Fallopian tube capture of chronic peritoneal dialysis catheters. Perit Dial Int. 1993;13(1):74-6.

5. Bradley AJ, Mamtora H, Pritchard N. Transvaginal leak of peritoneal dialysate demonstrated by CT peritoneography. Br J Radiol. 1997 Jun;70(834):652-3.

6. Ogunc G. Oygur N. Vaginal fistula: a new complication in CAPD. Perit Dial Int. 1995;15(1):84-5.

7. Diaz-Buxo J, Burgess P, Walker P. Peritoneovaginal fistula: unusual complication of peritoneal dialysis. Perit Dial Bull. 1983;3:1423.

8. Coward RA, Gokal R, Wise M, Mallick NP, Warrell D. Peritonitis associated with vaginal leakage of dialysis fluid in continuous ambulatory peritoneal dialysis. $\mathrm{Br}$ Med J (Clin Res Ed). 1982 May 22;284(6328):1529.

9. Wright CA, Silk MD. Is peritoneal vaginal fistula the main cause of fungal peritonitis in female CAPD patients. Perit Dial Int. 1984;4:51. 